\title{
The Sinusoid as the Longitudinal Profile in Backward-Wave Oscillators of Large Cross Sectional Area
}

\author{
Joaquim P. Leite Neto and Joaquim J. Barroso \\ Laboratório Associado de Plasma Instituto Nacional de Pesquisas Espaciais \\ Av. dos Astronautas, 1758 S. José dos Campos, SP, Brazil
}

Received on 11 December, 2003

\begin{abstract}
High-power generation in backward-wave oscillators (BWO) of large section requires that the beam electrons flowing close to the corrugated wall interact efficiently with surface waves supported by a periodic structure. Such waves are described by the superposition of slow-wave space harmonics of the operating mode. The present paper reports on design tools for BWOs operating in symmetric TM modes since these modes are able to perturb the axial velocity and electron density on rectilinear beams confined by an external magnetic field in slow-wave systems. Here we investigate whether a cylindrical guide with sinusoidally rippled wall can provide strong coupling between the guide surface waves and mildly relativistic $(\sim 500 \mathrm{keV})$ electron beams in the 8-9 GHz frequency range for BWOs of large diameter $(D \sim 3 \lambda)$. For this purpose, the characteristic equation of a sinusoidally corrugated structure is derived on the basis of the Rayleigh-Fourier method, whereby the field solution is represented by a single expansion of TM eigenmodes. From the dispersion diagrams thus obtained we infer the appropriate periodic length and ripple amplitude of the guiding structure that optimize the beam-wave interaction.
\end{abstract}

\section{Introduction}

The backward-wave oscillator (BWO) is a source of electromagnetic radiation in the centimeter and millimeter wavelength bands, with its capability as a high-power microwave source being demonstrated by the generation of $15 \mathrm{GW}$ output power at $3-\mathrm{cm}$ and $3 \mathrm{GW}$ at $8-\mathrm{mm}$ wavelengths [1]. In the simple classical description, the BWO consists of a spatially periodic waveguide into which a high-current electron beam (confined by a strong magnetic field) is injected to drive and interact with azimuthally symmetric transverse magnetic (TM) modes, as just these modes are able to perturb the axial velocity and electron density on rectilinear beams. In many works [2-8] the periodic structure, in general a sinusoidally rippled cylindrical guide, is merely displayed in its final configuration, with no account as to how the geometry of the structure has been synthesized aiming at its particular application. Adressing this basic question, here we present design tools for the synthesis of sinusoidal profiles from the technical goals for the BWO, namely, the operating frequency and the beam energy. The analysis develops on the basis of the Rayleigh-Fourier method, whereby the field solution is represented by a single expansion of TM space harmonics [10]. This method has been verified by some authors [11-14] as the one (among the least-squares and the integral methods) giving by far the best overall description of wave scattering from sinusoidal surfaces.

Consistent with the design requirements of enough slowing of the TM wave and strong coupling impedance, a sinusoidal profile is specifically synthesized for a $8.9 \mathrm{GHz}$
BWO driven by a $500 \mathrm{keV}$ electron beam. The RF method is also successfully applied in the analysis of a piecewise continuous profile [7] (with semicircles separated by rectangular grooves), which shows to yield a higher coupling impedance upon comparison with the sinusoidal profile.

\section{Dispersion relation of the cylin- drical waveguide with sinusoidally Rippled Wall}

The corrugated structure that we consider consists of a cylindrical waveguide with perfectly conducting wall of radius $R_{w}(z)$ sinusoidally rippled about the mean radius $R_{0}$ as that commonly employed in high-power BWO experiments [8], [9], i.e.,

$$
R_{w}(z)=R_{0}\left(1+\epsilon \cos \left(\frac{2 \pi}{d} z\right)\right)
$$

with $\epsilon$ and $d$ defining the amplitude and the period of corrugation, repectively. Due to the spatial periodicity of the structure along the axial coordinate $\mathrm{z}$, azimuthally symmetric TM electric fields in the cylindrical corrugated system $(r, \varphi, z)$ can be expanded in spatially harmonic series according to Floquet's theorem as

$$
E_{z}(r, z, t)=\sum_{n=-\infty}^{n=+\infty} A_{n} J_{0}\left(k_{\perp n} r\right) \exp \left(i k_{z n} z-i \omega t\right)
$$




$$
E_{r}(r, z, t)=-i \sum_{n=-\infty}^{n=+\infty} A_{n} \frac{k_{z n}}{k_{\perp n}} J_{1}\left(k_{\perp n} r\right) \exp \left(i k_{z n} z-i \omega t\right)
$$

where

$$
k_{z n}=k_{z 0}+\frac{2 \pi}{d} n, 0 \leq k_{z 0} \leq \frac{2 \pi}{d}
$$

denotes the longitudinal wavenumber of the nth space harmonic, which is related to the angular frequency $\omega$ and to the transverse wavenumber $k_{\perp n}$ through

$$
k_{\perp n}^{2}=\frac{\omega^{2}}{c^{2}}-k_{z n}^{2}
$$

Assuming $k_{z 0}$ as the reference propagation constant, the electric field phase shifts by $\exp \left(i k_{z n} d\right)$ in moving from $z$ to $z+d$ (at fixed $r$ and $\varphi$ ), with the determination of $k_{z 0}$ (and, hence, $k_{z n}$ ) as function of $\omega$ being the central problem of a slow-wave structure.

The dispersion equation determining the dependence of the reference wavenumber $k_{z 0}$ on the angular frequency follows from the boundary condition requiring that the component of the electric field tangential to the corrugated surface must vanish, i.e.,

$$
\left[E_{z}(r, z)+E_{r}(r, z) \frac{d R_{w}(z)}{d z}\right]_{r=R_{w}}=0
$$

Substituting (2)-(3) in (6) and expanding the resulting expression in a Fourier integral over $[0,2 \pi]$, we find an infinite system of algebraic equations for the amplitude coefficients $A_{n}$

$$
\sum_{n=-\infty}^{n=\infty} A_{n} C_{n m}\left(\omega, k_{z 0}, \epsilon, R_{0}, d\right)=0
$$

where

$$
C_{n m}=\left[1+\frac{2 \pi}{d}(n-m)\right] \frac{k_{z n}}{k_{\perp n}^{2}} \int_{0}^{d} d z J_{0}\left(k_{\perp n} R_{w}(z)\right) \operatorname{Cos}\left((n-m) \frac{2 \pi}{d} z\right)
$$

The existence of a nontrivial solution to the amplitudes of the space harmonics demands that the determinant of the homogeneous equations ( 7 ) be zero. Thus, setting

$$
\operatorname{det}\left\|C_{n m}\right\|=0
$$

yields the eingenvalue for at given corrugated parameters $\epsilon$, $R_{0}$ and $d$. Normalizing the dispersion relation and solving for $\omega R_{0} / c$ as function of $k_{z 0} d / \pi$ the periodic structure is described by two dimensionless parameters, $\epsilon$ and $d / R_{0}$.

Although (9) involves an infinite matrix, in practice we truncate the system to an adequate finite rank to obtain an approximation of the exact eigenvalue equation. We have verified that a $9 \times 9$ matrix gives (for the same structure) eigenvalues differing by $0.1 \%$ from those calculated with a higher order matrix. Therefore, in the ensuing calculations the rank of the matrix in (9) (with $n=m$ ) is truncated at 9 $(-4 \leq n \leq 4)$.

\section{Fundamentals of the backward- wave oscillator}

Linear beam relativistic microwave devices are based on the interaction of an electron beam and an electromagnetic field containing slow-wave components. Such a field can be realized in spatially periodic structures. Chiefly among these devices, is the backward-wave oscillator (BWO) with its essential characteristic of allowing electrical tuning, as the operating frequency is approximately determined by the synchronism condition: phase velocity of the wave $v_{p h}=$ electron velocity $v_{z}$. A BWO schematic is shown in Fig. 1 with the corresponding dispersion diagram, i.e., the dependence of the wave frequency on $k_{z} d$, the phase shift per period in a slow-wave structure of periodic length $d$ that supports a travelling wave of propagation constant $k_{z}$. The dashed line is the light line $\omega=k_{z} c$, which defines the boundary between regions of fast $\left(v_{p h}=\omega / k_{z}>c\right)$ and slow $\left(v_{p h}<c\right)$ waves. A passband refers to a frequency band ranging from the lower frequency (the cutoff frequency at points $k_{z}=0,2 \pi / d, \ldots$ ) and the upper frequency (at the $\pi$ point $\left.k_{z}=\pi / d, 3 \pi / d, \ldots\right)$. As we shall see, in a sinusoidally rippled structure the passband narrows as the ripple amplitude $\epsilon$ increases. The operating point is determined by the intersection of the dispersion curve with the beam line $\omega=k_{z} v_{z}$, corresponding to the condition of Cherenkov synchronism $v_{p h}=v_{z}$. With the presence of the driving electron beam, the BWO interaction takes place with the slow space-charge wave, $\omega=k_{z} v_{z}-\omega_{p}$ (where $\omega_{p}$ identifies the beam plasma frequency), a negative-energy wave whose growth decreases the kinetic energy of the beam. In the dispersion curve of the slow-wave structure, the BWO operates in regions where the group velocity $v_{g}=d \omega / d k_{z}$ is negative $\left(\pi / d<k_{z}<2 \pi / d\right.$, etc) and amplify backward waves, which propagate in the opposite direction as the electron beam and so provide an internal feedback mechanism. By changing the operating beam voltage (electron velocity) and/or the periodic length $d$ one can in principle move the point of intersection (of the beam Doppler line with the dispersion curve) to any point below the light line. 

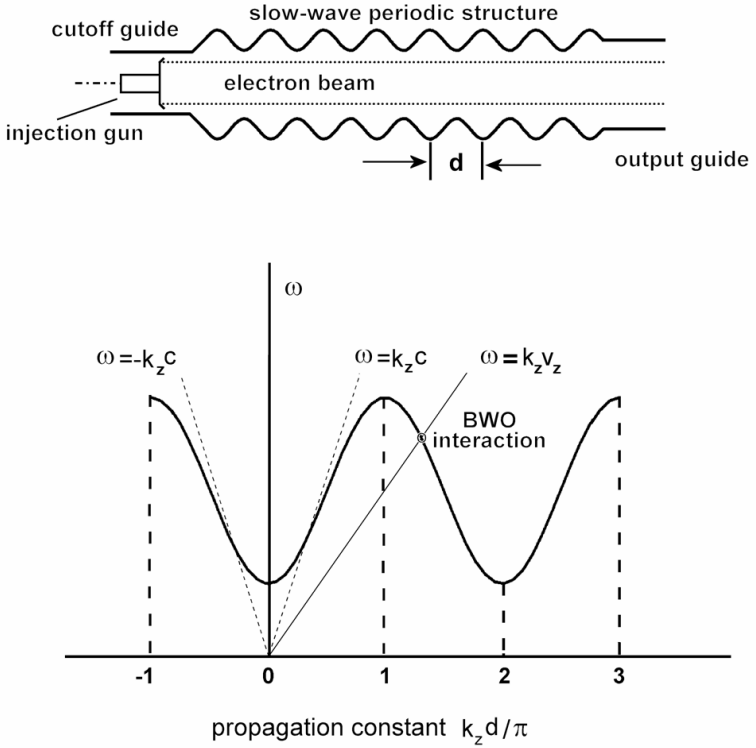

Figure 1. BWO schematics and dispersion diagram showing the first band.

\section{Synthesis and analysis of a sinusoi- dal profile}

The results which follow are primarily concerned with sinusoidal corrugations of small amplitude $\epsilon R_{0}<\lambda / 4$ in oversized cylindrical waveguides of average radius $R_{0}$ larger than the free-space wavelength $\lambda$ of the radiation. To assure single-mode operation in such oversized guides we keep the operating point close to the upper edge of the passband, the $\pi$ point. In this region, the smallness of the group velocity can make the diffraction $Q$ factor (of the mode of interest) $Q=\omega d / v_{g}$ large enough to favour excitation of the nominal mode against higher-order competing modes [7]. Then the practical problem we shall discuss relates to the synthesis of a sinusoidally rippled profile for a BWO designed to operate in the $\mathrm{TM}_{01}$ mode over the $8-9 \mathrm{GHz}$ frequency range and to be driven by an electron beam at the injection energy of $W_{b}=500 \mathrm{keV}$. Eigenfrequencies and phase velocities for $\mathrm{TM}_{01}$ mode operating close to the $\pi$ point are shown as function of the normalized ripple amplitude $\epsilon$ in Figs. 2 and 3, where the normalized periodic length $d / R_{0}$ is taken as parameter. In the plots, the beam energy is set at $W_{b}=(\gamma-1) m c^{2}=500 \mathrm{keV}$, with $\gamma=1.978$ which gives an electron velocity of $0.86 c$, where $c$ is the vacuum light speed. The requirement that the phase velocity of synchronous space harmonic should be slightly less than $0.86 c$ places a constraint on the corrugation period, namely $d /\left.R_{0}\right|_{\max } \leq 0.35$. Then taking $d / R_{0}=0.35$, for instance, and $0.03 \leq \epsilon \leq 0.07$ the associated frequencies lie within the specified 8-9 GHz frequency range (Fig. 2), yet from Fig. 3 the phase velocity remains above $0.85 c$ and, therefore, adequate slowing of the wave for interacting with the beam is barely achieved. On the other hand, fixing $d / R_{0}=0.30$ poses two constraints on $\epsilon$, the first of which requires $0.065 \leq \epsilon \leq 0.085$ so as to keep the design frequency in the 8-9 GHz range, whereas the second constraint demands $v_{p h} \leq 0.86 c$ to satisfy the synchronism condition. So we select from Fig. $3 \epsilon=0.07$ at $d / R_{0}=0.30$ to obtain $v_{p h}=0.82 c$ and the operating frequency of $8.6 \mathrm{GHz}$ (Fig. 2).

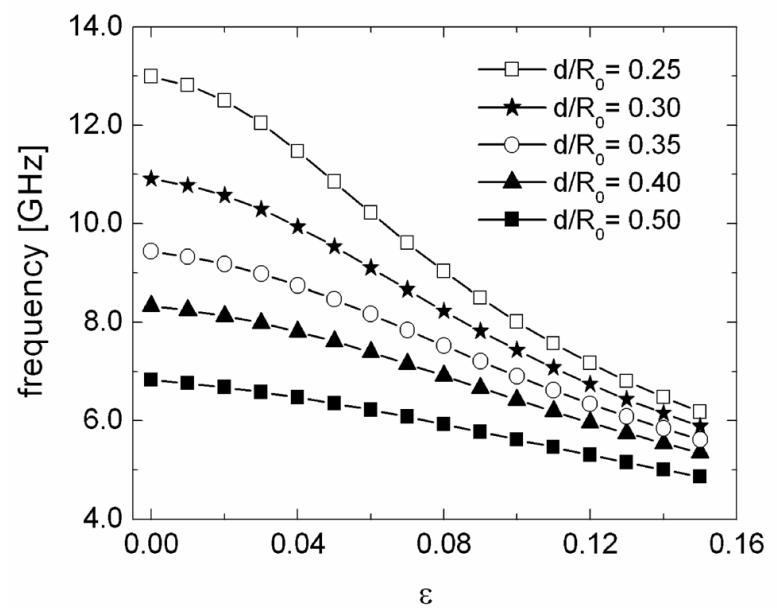

Figure 2 . $\mathrm{TM}_{01}$-mode upper cutoff frequencies $(\pi-$ point $)$ as function of the ripple amplitude $\epsilon$ with the normalized period $d / R_{0}$ as parameter.

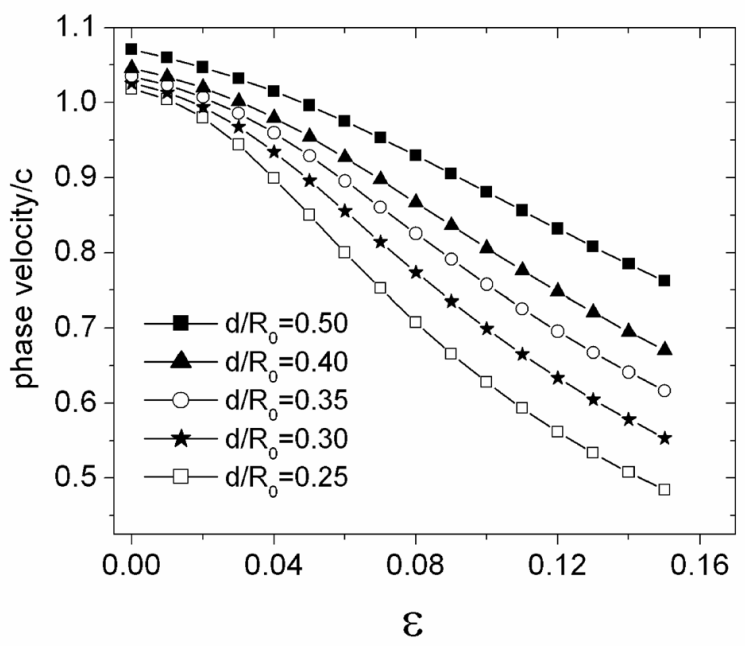

Figure 3. $\mathrm{TM}_{01}$-mode phase velocity (at the $\pi$-point) as function of the ripple amplitude $\epsilon$.

To put into perspective the attributes of the sinusoidal profile so far synthesized, we compare its performance with an experimentally tested periodic structure [7] designed to support surface waves in an overmoded $8.3 \mathrm{GHz}$ BWO operating at mildly relativistic beam energy $(500 \mathrm{keV})$. With periodic length $d=1.4 \mathrm{~cm}$, the longitudinal profile of such structure is composed of semicircles of radius 0.5 $\mathrm{cm}$ separated by rectangular grooves with optimized height $h=0.1 \mathrm{~cm}$. To compare the performance of the sinusoid, we set its minimal and maximal radii at $4.2 \mathrm{~cm}$ and 4.8 $\mathrm{cm}$ to fit the piecewise continuous profile as illustrated in Fig. 4. The $\mathrm{TM}_{01}$-mode dispersion diagrams for both profiles are compared in Fig. 5 covering the propagation constant(normalized) from 0 to $\pi$. We see that the curves are 
virtually coincident up to $k_{z o} d / \pi \simeq 0.8$, with the $\pi$-point frequencies differing by $2 \%$ at the upper edge of the passband intercepted by the Doppler beam line $\left(v_{z}=0.86 \mathrm{c}\right)$. But from the point of view of coupling impedance (defined as $E^{2} / 2 k_{z 0}^{2} P$ where $E$ is the average RF electric field at the beam position, and $P$ the power flow on the structure), it becomes apparent from Fig. 6, which shows at $z=0$ the radial dependence of the electric field, that the field strength near to the corrugated wall is substantially higher in the piecewise profile than in the sinusoidal counterpart.

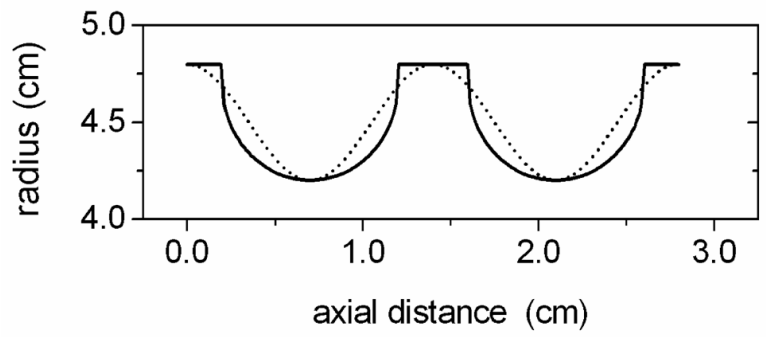

Figure 4. Sinusoidal $\left(R_{0}=4.5 \mathrm{~cm}, \epsilon=1 / 15, R_{\min }=4.2\right.$ $\mathrm{cm}, R_{\max }=4.8 \mathrm{~cm}$ ) and piecewise (semicircle radius $0.5 \mathrm{~cm}$ and rectangle height $0.1 \mathrm{~cm}$ ) profiles with periodic length $d=1.4 \mathrm{~cm}$.

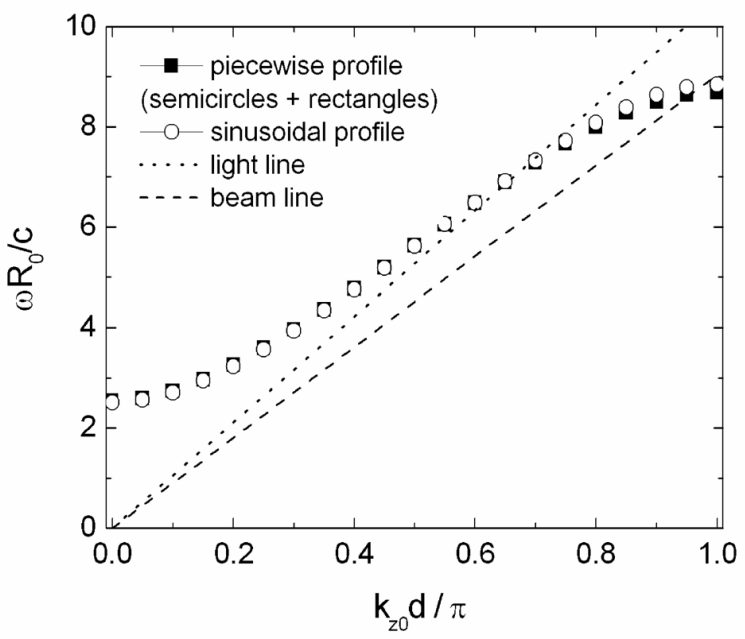

Figure 5. $\mathrm{TM}_{01}$-mode dispersion diagram corresponding to the sinusoidal and piecewise profiles in Fig. 4. Dashed and dotted lines are the light line and the $500 \mathrm{keV}$-beam Doppler line $\left(v_{z}=0.86 c\right)$.

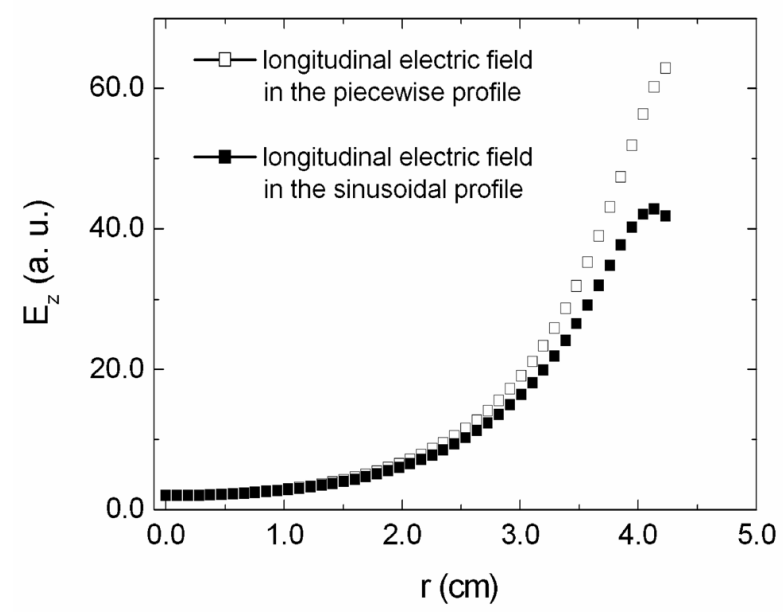

Figure 6. Radial dependence of the $\mathrm{TM}_{01}$-mode electric field on section $\mathrm{z}=0.0$ of sinusoidal and piecewise profiles shown in Fig. 4.

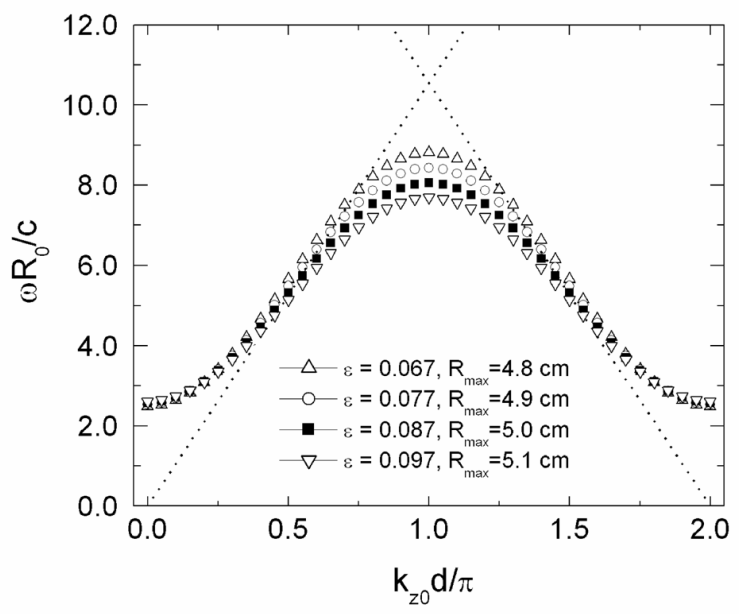

Figure 7. $\mathrm{TM}_{01}$-mode dispersion diagram for an oversized guide with longitudinal profile sinusoidally corrugated. The four curves are associated with distinct values of average radius $R_{0}$ and ripple amplitude $\epsilon$, but keeping in all cases $R_{\min }=4.2 \mathrm{~cm}$ and $d=1.4$ $\mathrm{cm}$. Dashed line is the light line.

Nevertheless, the field strength and the breadth of the passband (for the sinusoid) can be adjusted by proper variation of the corrugation parameters $\epsilon$ and $R_{0}$. To clarify this point, we show in Fig. 7 dispersion curves related to four sinusoidal profiles characterized by 1) $R_{0}=4.50 \mathrm{~cm}$, $\epsilon=0.0667$; 2) $R_{0}=4.55 \mathrm{~cm}, \epsilon=0.0769$; 3) $R_{0}=4.60$ $\mathrm{cm}, \epsilon=0.0869$; 4) $R_{0}=4.65 \mathrm{~cm}, \epsilon=0.0968$. Given by $R_{0}$, the lower cutoff frequency at $k_{z o} d=0$ is the same for all the curves, while the upper critical frequency, determined by $\epsilon$, assumes distinct values on each curve. We see that a dispersion curve flattens as $\epsilon$ increases, and so $d \omega / d k_{z}$ decreases accordingly. Because of this effect, and illustrated in Fig. 8, the field amplitude increases as the corrugation deepens. In fact, inspection of Fig. 8 indicates that the field intensity at $z=4.3 \mathrm{~cm}$ is four times as high for the deeper corrugation $(\epsilon=0.097)$, although the cojoined $\pi$ point frequency lies below $8.0 \mathrm{GHz}$ (Fig. 7). In view of this trade-off relation, the sinusoidal profile that meets the design specifications is identified by the corrugation parameters $R_{0}=4.50 \mathrm{~cm}, d=1.4 \mathrm{~cm}$, and $\epsilon=0.067$ which yield 
the critical frequency of $8.9 \mathrm{GHz}$ corresponding to the phase velocity of $0.82 c$ for the synchronous space harmonic.

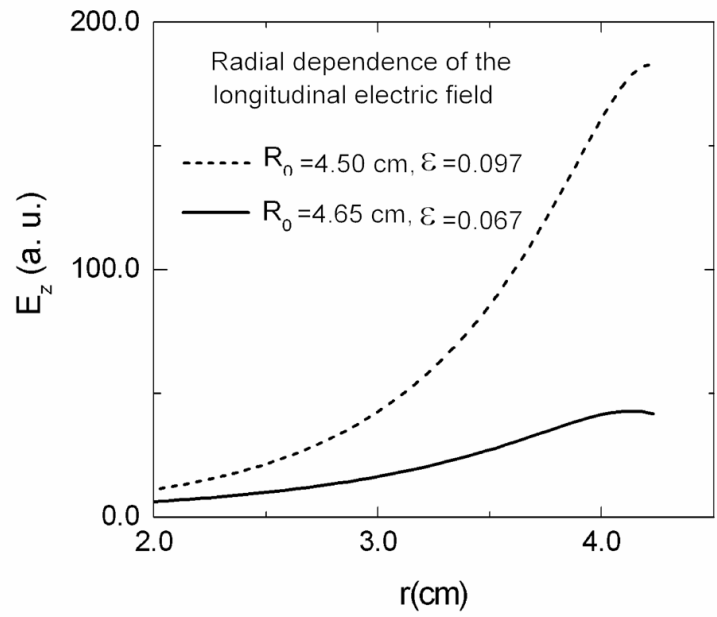

Figure 8. Radial dependence of the $\mathrm{TM}_{01}$-mode electric field (on section $\mathrm{z}=0.0$ ) in sinusoidally rippled waveguides.

To address the issue of mode competition concerning the symmetric modes $\mathrm{TM}_{01}, \mathrm{TM}_{02}$, and $\mathrm{TM}_{03}$, Fig. 9 plots (together with the $500-\mathrm{keV}$ beam line) their respective dispersion curves associated with the synthesized sinusoidal profile. We see that the beam line crosses the dispersion curves of the higher-order modes $\left(\mathrm{TM}_{02}, \mathrm{TM}_{03}\right)$ at frequencies above $10 \mathrm{GHz}$, significantly higher than the $8.9-\mathrm{GHz}$ frequency of the nominal $\mathrm{TM}_{01}$ mode. Well isolated and having the largest $Q$ factor, this makes the $\mathrm{TM}_{01}$ mode to be excited more readily than its nearest competitors.

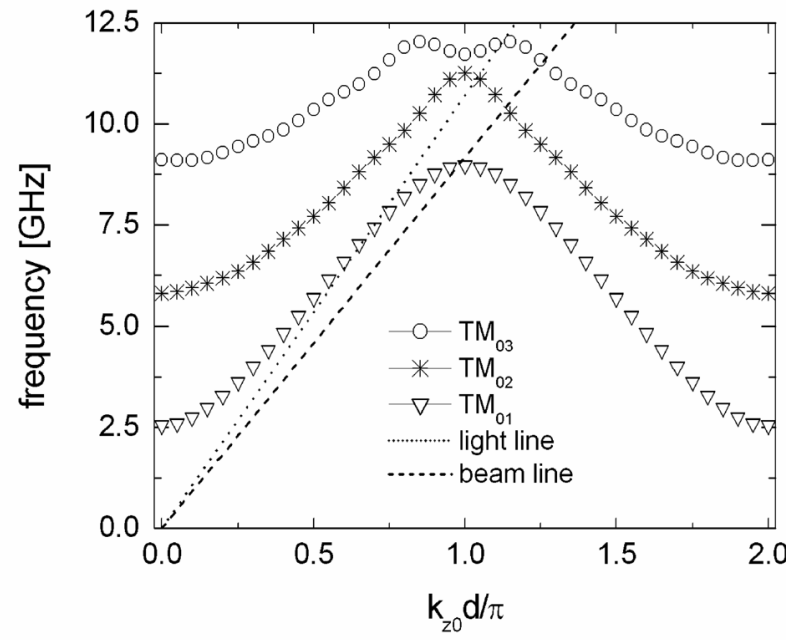

Figure 9. Calculated dispersion diagrams for low-order $\mathrm{TM}_{01}$ modes in the sinusoidally corrugated waveguide $\left(R_{0}=4.5 \mathrm{~cm}\right.$, $\epsilon=1 / 15, d=1.4 \mathrm{~cm}$ ) shown in Fig. 4. Light and beam lines are indicated by dotted and dashed lines.

Finally, to ascertain the validity of the numerical results obtained here from the Rayleigh-Fourier (RF) method, we compare in the Tab. 1, critical frequencies calculated from the RF method and those provided by a simulation computer code [7] used to optimize the piecewise profile combined with semicircles and rectangles. So we see that the
Rayleigh-Fourier results fit the WaveSym calculation wihin an accuracy better than $1 \%$.

TABLE 1. Critical frequencies (in GHz) calculated from the WaveSym code and from the RF method

\begin{tabular}{|c|c|c|}
\hline $\mathrm{h} / \mathrm{cm}$ & WaveSym Code & Rayleigh-Fourier Method \\
\hline 0.1 & 8.82 & 8.82 \\
\hline 0.2 & 8.30 & 8.28 \\
\hline 0.3 & 7.82 & 7.89 \\
\hline 0.4 & 7.33 & 7.40 \\
\hline
\end{tabular}

\section{Conclusions}

A systematic procedure has been given to synthesize, consistently with the required operating frequency and injection beam energy, an overmoded sinusoidally corrugated waveguide with application as a slow-wave structure on backward-wave oscillators. The corrugation parameters are selected so that the waveguide when driven by a $500-\mathrm{keV}$ electron beam operates close to the upper cutoff frequency on the first space harmonic of the lower order $\mathrm{TM}_{01}$ mode. Consistent with single-mode operation, the periodic structure here designed supports a surface wave that is synchronous with a 500-keV electron beam and simultaneously exhibits a large coupling impedance, which is a measure of the RF field strength at the beam position. The surface wave is crucial to avoiding mode competition, while large coupling impedance is important for high-efficiency interaction.

The mathematical formalism used in the paper relies on the Rayleigh-Fourier method, which has shown excellent performance even when used on the analysis of a piecewise continuous profile made from a combination of semicircles and rectangles.

\section{References}

[1] A. Vlasov, V. A. Cherepenin, and V. N. Kornienko, IEEE Trans. Plasma Sci. 24, 870 (1996).

[2] L. D. Moreland, E. Schamiloglu, R.W. Lemke, S. D. Korovin, V. V. Rostov, A. M. Roitman, K. J. Hendricks, and T. A. Spencer, IEEE Trans. Plasma Sci. 22, 554 (1994).

[3] S. D. Korovin, G. A. Mesyatz, I. V. Pegel, S. D. Polevin, and V. P. Tarakanov, IEEE Trans. Plasma Sci. 28, 485 (2000).

[4] K. Ogura, K. Minami, Md. M. Ali, Y. Kan, T. Nomura, Y. Aiba, A. Sugawara, and T. Watanabe, J. Phys. Soc. Japan, 61, 3966 (1992).

[5] H. Guo, Y. Carmel, W. R. Lou, L. Chen, J. Rodgers, D. K. Abe, A. Bromborsky, W. Destler, and V. L. Granatstein, IEEE Trans. Microwave Theory Tech. 40, 2086 (1992).

[6] W. Main, Y. Carmel, K. Ogura, J. Weaver, G. S. Nusinovich, S. Kobayashi, J. P. Tate, J. Rodgers, A. Bromborsky, S. Watanabe, M. R. Amin, K. Minami, W. W. Destler, and V. L. Granatstein, IEEE Trans. Plasma Sci.22, 566 (1994). 
[7] A. N. Vlasov, A. G. Shkvarunets, J. C. Rodgers, Y. Carmel, T. M. Antonsen Jr., T. M. Abuelfadl, D. Lingze, V. A. Cherepenin, G. S. Nusinovich, M. Botton, and V. L. Granatstein, IEEE Trans. Plasma Sci.28, 550 (2000).

[8] A. I. Klimov, S. D. Korovin, V. V. Rostov, M. R. Ulmaskulov, V. G. Shpak, S. A. Shunailov, and M. I. Yalandin, IEEE Trans. Plasma Sci. 30, 1120 (2002).

[9] K. Minami, M. Saito, Y. Choyal, K. P. Maheshwari, and V. L. Granatstein, IEEE Trans. Plasma Sci. 30, 1134 (2002).

[10] J. J. Barroso, J. P. Leite Neto, and K.G. Kostov, IEEE Trans.
Plasma Sci. 31, 752 (2003).

[11] A. Wirgin, Opt. Acta, 27, 1671 (1980).

[12] J. P. Hugonin, R. Petit, and M. Cadilhac, J. Opt. Soc. Am. 71, 593 (1981).

[13] M. Neviere, M. Cadilhac, and R. Petit, IEEE Trans. Antennas Propagat. 21, 37 (1973).

[14] P. M. Van den Berg and J. T. Fokkema, J. Opt. Soc. Am. 69, 27 (1979). 